\title{
Quantum Ontology in the Light of Gauge Theories
}

\author{
Gabriel Catren ${ }^{\mathrm{a}}$ \\ ${ }^{a}$ Department of Philosophy, University of Buenos Aires, Argentina
}

\begin{abstract}
We propose the conjecture according to which the fact that quantum mechanics does not admit sharp value attributions to both members of a complementary pair of observables can be understood in the light of the symplectic reduction of phase space in constrained Hamiltonian systems. In order to unpack this claim, we propose a quantum ontology based on two independent postulates, namely the phase postulate and the quantum postulate. The phase postulate generalizes the gauge correspondence between first-class constraints and gauge transformations to the observables of unconstrained Hamiltonian systems. The quantum postulate specifies the relationship between the numerical values of the observables that permit us to individualize a physical system and the symmetry transformations generated by the operators associated to these observables. We argue that the quantum postulate and the phase postulate are formally implemented by the two independent stages of the geometric quantization of a symplectic manifold, namely the prequantization formalism and the election of a polarization of pre-quantum states respectively.
\end{abstract}

Keywords: quantum mechanics, gauge theories, geometric quantization, symplectic reduction

\section{Introduction}

In this article we consider quantum mechanics in the light of a fundamental idea coming from gauge theories, namely that first-class constraints induce gauge transformations [25]. In gauge theories, the symmetries defined by gauge transformations reduce the amount of observable (or gauge invariant) information that is necessary to completely describe a physical system.

Email address: gabrielcatren@gmail.com (Gabriel Catren) 
More precisely, the symplectic reduction defined by the first-class constraints of the theory permits us to pass from the original constrained phase space, which contains both physical and non-physical degrees of freedom, to the socalled reduced phase space, which only describes gauge invariant quantities $[9,25,32]$. In this way, a constrained Hamiltonian system of $2 n$ canonical variables and $k$ first-class constraints can be reduced to an unconstrained Hamiltonian system of $2(n-k)$ physical canonical variables. The heuristic conjecture of the present article is that a similar idea can be used for explaining the fact that both members of a complementary pair of observables cannot be sharp objective properties of the same quantum system in accordance with Heisenberg uncertainty principle. In other terms, our main claim is that the reduction in the number of observables that are necessary to completely describe a physical system from $2 n$ classical observables (e.g. $q$ and $p$ ) to $n$ quantum observables (e.g. $q$ or $p$ ) can be understood in the light of the symplectic reduction of phase space in gauge theories. In order to generalize to unconstrained Hamiltonian systems (what we shall call from now on) the "gauge correspondence" between first-class constraints and gauge transformations, we argue that there exists a universal symmetry acting on the phase space of every Hamiltonian system, be it constrained or not. The action of this universal symmetry defines a "projection" from the $2 n$ classical observables $q$ and $p$ to the $n$ quantum observables $q$ or $p$ (or a mixture of both in accordance with Heisenberg uncertainty principle). In order to distinguish this universal symmetry and the corresponding symmetry transformations from gauge symmetries and gauge transformations - which are only present in the framework of constrained Hamiltonian systems-, we call the former phase symmetry and phase transformations respectively.

In order to ascertain the conceptual meaning of phase symmetry, we show that the existence of this universal symmetry is a direct consequence of a particular ontology of physical systems. This quantum ontology can be understood as an extension of what we shall call pre-ontology. According to the latter, what we shall call an object is a "multifaceted" structure (1) that has different aspects, profiles, or faces (which will be called from now on phases); and (2) that is defined by a set of invariant objective properties that permit observers to recognize the object in spite of its multiple phases. We argue that the quantum ontology can be obtained by adding two postulates to the pre-ontology, namely the phase postulate and the quantum postulate. 
The phase postulate generalizes the gauge correspondence between firstclass constraints and gauge transformations to the relation between objective properties and phase transformations. More precisely, the phase postulate states that the operators associated to the objective properties of an object generate its phase transformations. It can be shown that, from a conceptual point of view, Heisenberg uncertainty principle is a natural consequence of the following two claims: (1) the operators associated to the objective properties of a physical system generate its phase transformations (phase postulate), and (2) the objective properties must be invariant under these phase transformations. This last assertion is just a particular implementation of the standard relationship between objectivity and invariance under a symmetry group $[4,6,16,17,18,19,20,31,40]$.

In turn, the quantum postulate establishes a faithful correspondence between the numerical values of the objective properties of an object and the phase transformations generated by their associated operators. This postulate implies that the particular numerical value of an objective property like a momentum $p$, far from being intrinsically related to the velocity $\dot{q}$ (i.e. to the transformation of $q$ in time), faithfully specifies how the corresponding object virtually transforms under the phase transformations generated by the operator associated to $p$ (i.e. the translations in $q$ ). We finish arguing that the quantum postulate and the phase postulate are formally implemented by the two independent stages of the geometric quantization formalism, namely (1) the prequantization of a sympletic manifold, and (2) the definition of a polarization respectively [7, 33, 37, 42].

This article continues the work started in Refs.[11, 12]. While the phase postulate was introduced in Ref.[12], a first formulation of the quantum postulate was proposed in Ref.[11]. The construction of a quantum ontology compatible with these postulates was started in Ref.[11]. The main contributions of the present article are (1) the claim according to which the phase postulate generalizes the gauge correspondence between first-class constraints and gauge transformations to unconstrained Hamiltonian systems, and (2) a reformulation of the quantum postulate in terms of the relationship between the numerical values of the objective properties and the corresponding phase transformations. ${ }^{1}$

\footnotetext{
${ }^{1}$ In Ref.[11] the quantum postulate is formulated in the framework of the momentummap formalism for Hamiltonian actions on symplectic manifolds.
} 
The organization of the paper is as follows. In Section II, we revisit the relevant aspects of symplectic geometry and constrained Hamiltonian systems. In Section III, we introduce the pre-ontology of physical objects. In Sections IV and V, we introduce the phase postulate and the quantum postulate respectively. In Section VI, we revisit the difference between momenta $p$ and velocities $\dot{q}$ in the light of the proposed postulates. In Section VII, we analyze the relationship between this twofold conceptual reconstruction of quantum mechanics on the one hand and the geometric quantization of a symplectic manifold on the other. In the final section we summarize the obtained results and its formal consequences.

\section{Symplectic Geometry and Constrained Hamiltonian Systems}

From a geometric point of view, the phase space of a classical Hamiltonian system of $n$ degrees of freedom is a $2 n$-dimensional symplectic manifold $(P, \omega)$. A symplectic manifold is a manifold $P$ endowed with a 2 -form $\omega$ (called symplectic structure) that is closed $(d \omega=0)$ and non-degenerate $\left(i_{v} \omega=0 \Leftrightarrow v=0\right)$, where $d$ is the exterior differential on differential forms on $P$

$$
d: \Omega^{k}(P) \rightarrow \Omega^{k+1}(P), \quad d^{2}=0,
$$

and $i_{v} \omega$ denotes the contraction of the differential form $\omega$ with the vector field $v[1,2]$. Darboux's theorem states that there always exist local coordinate systems $\left(q^{i}, p_{i}\right)$ such that $\omega$ locally takes the form $\omega=\sum_{i} d p_{i} \wedge d q^{i}$ (for the sake of simplicity we shall omit from now on the indices $i$ ). In the simplest case, the phase space of a classical system is given by the cotangent bundle $P=T^{*} Q \stackrel{\pi}{\rightarrow} Q$ over the configuration space $Q$. The symplectic 2 -form of a cotangent bundle can be obtained from a canonical 1-form $\theta$ on $P$ by means of the expression $\omega=d \theta$ (which means that $\omega$ is globally exact in this particular case). ${ }^{2}$

\footnotetext{
${ }^{2}$ The canonical 1-form $\theta$ can be defined as follows. For any vector $v \in T_{x}\left(T^{*} Q\right)$, the projection $\pi: T^{*} Q \rightarrow Q$ defines the pushforward $\pi_{*} v \in T_{\pi(x)} Q$. On the other hand, the point $x \in T^{*} Q$ defines a pair $\left(q_{x}, p_{x}\right)$, where by definition the 1-form $p_{x}$ acts on vectors in $T_{\pi(x)} Q$ (i.e. $\left.p_{x} \in T_{\pi(x)}^{*} Q\right)$. This means that one can contract the 1-form $p_{x}$ and the vector $\pi_{*} v$. One can then define the canonical 1-form $\theta$ on $P=T^{*} Q$ by means of the expression $i_{v} \theta(x)=i_{\pi_{*} v} p_{x}$. In local coordinates $(q, p)$ on $T^{*} Q$, the canonical 1-form is $\theta=-p d q$.
} 
The 2-form $\omega$ of a symplectic manifold defines a correspondence

$$
\begin{aligned}
\tau: \mathcal{C}^{\infty}(P) & \rightarrow \mathcal{H}_{P} \subset T P \\
f & \mapsto v_{f}
\end{aligned}
$$

between real smooth functions $f$ (classical observables) and the so-called Hamiltonian vector fields $v_{f}$ (which we shall also call from now on classical operators). This correspondence is defined by means of the map

$$
\begin{aligned}
T^{*} P & \rightarrow T P \\
d f & \mapsto v_{f}
\end{aligned}
$$

given by the following expression

$$
i_{v_{f}} \omega=d f
$$

In $\mathbb{R}^{2}$, the Hamiltonian vector field associated to an observable $f \in$ $\mathcal{C}^{\infty}(P)$ takes the simple form

$$
v_{f}=\frac{\partial f}{\partial p} \frac{\partial}{\partial q}-\frac{\partial f}{\partial q} \frac{\partial}{\partial p} .
$$

The correspondence (1) defines a Poisson structure on the space of classical observables $\mathcal{C}^{\infty}(P)$ through the Poisson bracket ${ }^{3}$ :

$$
\{f, g\}=v_{g}(f) \in \mathcal{C}^{\infty}(P) .
$$

It can be shown that the Poisson bracket is a Lie algebra, which means that it satisfies bilinearity, skew-symmetry $(\{f, g\}=-\{g, f\})$ and the Jacobi identity $(\{f,\{g, h\}\}+\{h,\{f, g\}\}+\{g,\{h, f\}\}=0)$. The set of classical observables $\mathcal{C}^{\infty}(P)$ endowed with the Poisson structure defines a Poisson algebra. The Jacobi identity can also be expressed as

$$
\left[v_{f}, v_{g}\right]=v_{\{f, g\}} \cdot
$$

This means that the map $f \mapsto v_{f}$ is a Lie algebra homomorphism from the Poisson algebra $\mathcal{C}^{\infty}(P)$ to the Lie algebra of Hamiltonian vector fields $\mathcal{H}_{P}$. Moreover, the Poisson bracket structure and the commutative algebra

\footnotetext{
${ }^{3}$ The Poisson bracket can also be defined by means of the expression $\{f, g\}=i_{v_{g}} i_{v_{f}} \omega$. Indeed, $i_{v_{g}} i_{v_{f}} \omega=i_{v_{g}}(d f)=v_{g}(f)=\{f, g\}$.
} 
structure associated to the pointwise multiplication of functions in $\mathcal{C}^{\infty}(P)$ are related by means of the Leibnitz rule:

$$
v_{h}(f g)=\{f g, h\}=g\{f, h\}+f\{g, h\}=g v_{h}(f)+f v_{h}(g) .
$$

Hence, the Poisson structure makes $\mathcal{C}^{\infty}(P)$ into a Lie algebra where the Poisson bracket acts as a derivation in each argument. Indeed, it is worth noting that $\{f, g\}=v_{g}(f)$ is by definition the Lie derivative $\mathcal{L}_{v_{g}} f$ of $f$ in the direction defined by the vector field $v_{g}$.

The classical operators $v_{f}$ generate symplectic diffeomorphisms - or canonical transformations - of phase space $(P, \omega)$, that is to say diffeomorphisms of $P$ that preserve the symplectic structure $\omega$. Indeed, the Lie derivative of $\omega$ along a Hamiltonian vector field $v_{f}$ is identically zero:

$$
\mathcal{L}_{v_{f}} \omega=\left(d i_{v_{f}}+i_{v_{f}} d\right) \omega=d^{2} f=0
$$

where we used (3) and $d \omega=0$. We can thus say that a classical observable $f$ induces an action on physical states in $(P, \omega)$ given by the symplectic diffeomorphisms generated by its associated classical operator $v_{f}$. These considerations show that the use of operators acting on physical states, far from being introduced only in the framework of quantum mechanics, is already an essential feature of classical mechanics. In other terms, we could say that the canonical quantization condition

$$
\text { Poisson brackets (of observables) } \rightsquigarrow \text { Commmutators (of operators) }
$$

is already realized in classical mechanics by the Lie algebra homomorphism $\mathcal{C}^{\infty}(P) \rightarrow \mathcal{H}_{P}$. Moreover, two classical operators $v_{f}$ and $v_{g}$ do not necessarily commute. As we shall see below, the fact that the classical correspondence (1) between observables and classical operators is not entirely satisfactory justifies the necessity of extending the classical operators to quantum operators. These remarks have a consequence of fundamental importance, namely that in order to understand the differences between classical and quantum mechanics we do not have to compare the non-commutative algebra of quantum operators with the commutative ring structure of classical observables $\mathcal{C}^{\infty}(P)$ (relative to pointwise multiplication), but rather with the Lie algebra of classical operators $\mathcal{H}_{P}$.

In the rest of this section, we shall briefly describe the theory of classical constrained Hamiltonian systems (or gauge systems). A constrained 
Hamiltonian system is a Hamiltonian system $(P, \omega)$ endowed with a set of $k$ first-class constraints $G_{a}(q, p) \in \mathcal{C}^{\infty}(P)$, with $a=1, \ldots, k$ [25]. First-class constraints are real smooth functions on the phase space $P$ that are in involution, that is to say that form a closed Poisson algebra:

$$
\left\{G_{a}, G_{b}\right\}=f_{a b}^{c} G_{c}
$$

where $f_{a b}^{c}$ are functions on $P$ called structure functions. First-class constraints play a twofold role. On the one hand, they define the constraint surface $\Sigma \subset P$ by means of the constraint equations:

$$
G_{a}(q, p)=0, \quad a=1, \ldots, k .
$$

The constraint surface $\Sigma$ is a submanifold of the phase space $P$ of dimension $2 n-k$. The restriction of the symplectic form $\omega$ to $\Sigma$ is closed but not non-degenerate. In other terms, $\left.\omega\right|_{\Sigma}=\iota^{*} \omega$ is a pre-symplectic form, where $\iota: \Sigma \hookrightarrow P$ is the inclusion map. Hence, the constraint surface $\Sigma$ is a pre-symplectic submanifold of $P$. In fact, the Hamiltonian vector fields $v_{a}$ defined by the constraints $G_{a}$ satisfy $\left.i_{v_{a}} \omega\right|_{\Sigma}=0$. This means that $v_{a}$ are null vector fields of the pre-symplectic form $\left.\omega\right|_{\Sigma}$ (see Ref.[25], chap. 2). The integral lines of the null vector fields $v_{a}$-i.e. the so-called gauge orbitsdefine a null foliation of the constraint surface $\Sigma$. The first-class constraints $G_{a}(q, p)$ induce infinitesimal gauge transformations of classical observables $f(q, p)$ along the gauge orbits by means of the expression ${ }^{4}$ :

$$
\mathcal{L}_{v_{a}} f=v_{a}(f)=\left\{f, G_{a}\right\}
$$

In this way, first-class constraints $G_{a}(q, p)$ induce an action on observablesand therefore on states - by means of theirs associated classical operators $v_{a}$. In well-behaved circumstances, the null foliation defined by the gauge orbits is fibrating, which means that there exists a projection map $\pi: \Sigma \rightarrow$ $\left(P_{r e d}, \omega_{r e d}\right)$ over the quotient space $\left(P_{r e d}, \omega_{r e d}\right)$. This quotient space is a symplectic manifold called reduced phase space, where the reduced symplectic form $\omega_{\text {red }}$ satisfies the expression $\pi^{*} \omega_{\text {red }}=\iota^{*} \omega$, with $\iota: \Sigma \hookrightarrow P$.

\footnotetext{
${ }^{4}$ In the rest of this article we shall use the following terminology: the expression "an observable $f$ induces a transformation" will sometimes be used as an abbreviation for the longer expression "the operator $v_{f}$ associated to an observable $f$ generates a transformation".
} 
It is worth noting that the involution condition (7) is the Frobenius' integrability condition for distributions [9]. The fact that first-class constraints are in involution means that the gauge orbits induced by one constraint remain in the constraint surface defined by the others. For instance, the gauge transformation of the constraint $G_{a}$ generated by the constraint $G_{b}$ is given by the Poisson bracket $\mathcal{L}_{v_{G_{b}}} G_{a}=\left\{G_{a}, G_{b}\right\}=f_{a b}^{c} G_{c} \approx 0$, where the weak equality " $\approx$ " means that the left side of the equation is equal to zero only on the constraint surface $\Sigma$ defined by the constraints. Hence, the involution condition (7) guarantees that the constraint $G_{a}$ is invariant on the constraint surface $\Sigma$ under the gauge transformation generated by $G_{b}$. This means that the gauge orbits generated by $G_{b}$ remain in the surface $G_{a}=0$. It is also worth remarking that the involution condition (7) can also be interpreted in the following terms. Two constraints $G_{a}(q, p)=0$ and $G_{b}(q, p)=0$ are said to be "compatible" — which means that they can be used to define the same constraint surface - only if they "commute" weakly, i.e. only if $\left\{G_{a}, G_{b}\right\}=f_{a b}^{c} G_{c} \approx 0$. Reciprocally, we could say that (weak) Poisson non-commutativity defines the obstruction to the compatibility of different constraints. As we shall see in what follows, the fact that observables that do not have vanishing Poisson bracket cannot be sharp objective properties of the same quantum system admits an analogous interpretation.

According to the usual understanding of gauge theories, the gauge transformations induced by first-class constraints are transformations of the coordinate systems that do not modify the physical states. Hence, each element $x \in P_{\text {red }}$ in the reduced phase space of the theory is an equivalence class composed of all the different coordinate representations of the same physical state. The so-called gauge observables of the theory are the functions on $P_{\text {red }}$. Since in general it is difficult to construct the reduced phase space $P_{\text {red }}$ of a theory, it is important to know how to recover the observable information from the functions on the original phase space $(P, \omega)$. To do so, it is necessary to characterize the functions on $P_{r e d}$ in terms of functions on $P$. This can be done by firstly remarking that the functions on $P_{\text {red }}$ can be identified to the gauge invariant functions on the constraint surface $\Sigma$. Indeed, functions $f \in \mathcal{C}^{\infty}(\Sigma)$ that are not invariant along the gauge orbits generated by the constraints $G_{a}$ do not define functions on the quotient space $P_{\text {red }}$. The second step towards defining gauge observables in terms of functions on the original phase space $P$ is to characterize the functions on 
the constraint surface $\Sigma$ in terms of functions on $P$. This can be done by defining functions on $\Sigma$ as equivalence classes of functions on $P$, where two functions $f, g \in \mathcal{C}^{\infty}(P)$ belong to the same equivalence class if they differ in a function that vanishes on $\Sigma$, i.e. if $f-g=f^{a} G_{a} \approx 0$ (where the coefficients $f^{a}$ are phase space functions). In other terms, $\mathcal{C}^{\infty}(\Sigma)=\mathcal{C}^{\infty}(P) / \mathcal{I}_{\Sigma}$, where $\mathcal{I}_{\Sigma}$ is the ideal of functions vanishing on $\Sigma$. Thanks to this two-step characterization of functions on $P_{\text {red }}$ in term of functions on $P$, the gauge observables of the theory can be recovered by means of a cohomological algorithm, namely the so-called BRST cohomology [25]. The nilpotent BRST operator $\Omega_{B R S T}$ of this cohomology theory is defined in such a way that its zero degree cohomology $H^{0}\left(\Omega_{B R S T}\right)$ coincides with the set of observables of the system. This means that the operator $\Omega_{B R S T}$ encodes both the restriction to the constraint surface $\Sigma$ and the projection to the reduced phase space $P_{\text {red }}{ }^{5}$

By construction, the gauge observables do not distinguish between states belonging to the same gauge orbit. However, this does not mean that the theory is "incomplete", that is to say that there could exist hypothetical "hidden gauge variables" capable of physically distinguishing between gauge equivalent states. Gauge observables cannot distinguish between states belonging to the same gauge orbit because these states are just different coordinate representations of the same physical state. If one did not assume that states in a gauge orbit are physically equivalent, then the theory would be indeterministic [21]. As we shall claim below, the situation seems to be analogous in quantum mechanics. According to the proposed interpretative framework, it is not the case that quantum mechanics is incomplete, but rather that the different values of the coordinate $q$ of a system characterized by a sharp value of the momentum $p$ are phase equivalent, which means that they can be related by means of phase transformations. In other terms, the different values of $q$ of a system characterized by a sharp value of $p$ belong to the same phase orbit.

\footnotetext{
${ }^{5}$ Conceptual and geometric interpretations of the BRST construction in the framework of Yang-Mills theory can be found in Refs.[13, 14] respectively.
} 


\section{Pre-ontology of physical objects}

In this section we introduce what we shall call a "pre-ontology" of physical objects. This pre-ontology is intended to provide a notion of symmetry capable of explaining the fact that the quantum description of every Hamiltonian system does not admit sharp value attributions to both members of a complementary pair of observables. The fact that every Hamiltonian system must be described in quantum mechanical terms (even if their behavior can be approximated by classical mechanics under certain conditions) implies that the notion of symmetry that we need cannot depend on the particular characteristics of specific physical systems, such as gauge symmetries or particular physical symmetries (e.g. systems with spherical invariance, etc.).

Before characterizing the pre-ontology, we shall describe the heuristic idea that will lead us to the required notion of symmetry. Classical observables $f \in \mathcal{C}^{\infty}(P)$ play a twofold role in mechanics. Firstly, they can be evaluated on states $x \in P$ and used to individualize them. Indeed, the properties $f_{i}(x)$ defined by a complete set of classical observables suffice for identifying any state $x \in P .{ }^{6}$ Secondly, classical observables induce symplectic diffeomorphisms of phase space $(P, \omega)$ through the action generated by their associated classical operators $v_{f}$ (Hamiltonian vector fields). It is worth stressing that this correspondence between observables and classical operators (which is defined by the symplectic structure of phase space) is a fundamental feature of classical mechanics that has remained for the moment uninterpreted. The twofold role played by classical observables means that any Hamiltonian system can be individualized by specifying the numerical values defined by a complete set of classical observables, which in turn induce symplectic diffeomorphisms of phase space. This means that the very individualization of a Hamiltonian system naturally entails a notion of transformation. The pre-ontology that we shall propose and its quantum extension are intended to interpret these transformations as symmetry transformations of the corresponding system. These symmetry transformationsthat we shall call phase transformations-will permit us to define a universal (i.e. valuable for any Hamiltonian system, be it constrained or not) notion

\footnotetext{
${ }^{6} \mathrm{~A}$ set $\left\{f_{i}\right\}_{i} \subset \mathcal{C}^{\infty}(P)$ is a complete set of classical observables if and only if every other function $g$ that satisfies $\left\{f_{i}, g\right\}=0$ for all $f_{i}$ is necessarily constant.
} 
of symmetry capable of explaining the fact that the complete description of a quantum system only requires $n$ canonical variables (instead of the $2 n$ canonical variables required in classical mechanics).

The pre-ontology is based on the definition according to which the term object will denote a multifaceted structure characterized by a set of invariant objective properties. Firstly, this means that an object, far from being a point-like or structureless entity, is a configuration that has different "perspectival" aspects, profiles, faces, or (as we shall call them from now on) phases. Hence, we could say that an object is a multifaceted superposition of phases. The phase that an object exposes to a hypothetical second object (which we could call the observer) depends on the relative orientation between the two objects. In order to "observe" different phases of the object, it is necessary to perform either an "active" transformation of the object or, equivalently, a "passive" transformation of the observer's position. The transformations that permit the observer to observe the different phases of an object will be called phase transformations. A set of phases connected by means of a one-parameter family of phase transformations will be called phase orbit. The set of phase transformations that generate all the object's phase orbits define what we shall call the phase group of the object. These definitions can be illustrated by considering an ordinary example of a multifaceted structure, like for instance a die. A rotation of a die around one of its main axes is a phase transformation that permits an observer to observe the corresponding sequence of four numbers. By definition, these four numbers belong to the same phase orbit.

Besides having different phase orbits, an object is a configuration characterized by a set of objective properties that permit observers to identify the object in spite of the perspectival variation of its phases. More precisely, an object will be individualized by a set of values $\left\{f_{\alpha}^{1}, \ldots, f_{\rho}^{n}\right\}$ defined by a set of observables $\left\{f^{1}, \ldots, f^{n}\right\}$, where $f_{\mu}^{i}$ denotes a particular value of the observable $f^{i}$. Since phase transformations interchange different phases of the same object, the objective properties $\left\{f_{\alpha}^{1}, \ldots, f_{\rho}^{n}\right\}$ that characterize the object as such must be invariant under the object's phase group. In this way, the pre-ontology provides a particular realization of the standard group-theoretical relationship between objectivity on the one hand and invariance under symmetry transformations on the other (different analysis of this correspondence can be found in Refs. $[4,6,19,20,23,30,31,40])$. 
However, it is worth stressing that the different elements of a phase orbit should not be understood as different coordinate representations of the same physical configuration as it is the case in gauge theories, but rather as noninvariant "perspectival" components of the object's intrinsic multifaceted structure. Hence, it is necessary to establish a distinction between the usual epistemological realization of the correspondence objectivity-invariance and the ontological realization. The fact that a geometric object (such as a vector field or a tensor field) admits different coordinate representations does not mean that coordinate transformations could permit an observer to gain access to different intrinsic profiles of the geometric object. On the contrary, the different phases of an object that are interchanged by the corresponding phase transformations belong to the intrinsic multifaceted structure of the object. Even if its phases are not invariant under phase transformations, they are different intrinsic profiles of the same object.

It is worth stressing that phase transformations define a universal notion of invariance that stems from the definition according to which every object is, independently of its particular characteristics, a multifaceted structure. Hence, it is important not to confuse the universal phase symmetry with the possible particular symmetries of specific systems (like for instance the symmetries of snow crystals, regular polygons, or systems with spherical invariance). In order to clarify this point, let's consider for instance two dice of six phases: a normal die A (whose phases are numbered from one to six) and a die B such that the same number is printed on opposite phases (let's say the numbers 1, 3 and 5 for each pair of opposite phases). According to the pre-ontology, the objective properties of both dice have to be invariant under the corresponding phase groups. Besides this universal symmetry, the die B has an additional particular symmetry, namely a symmetry under rotations of $n 180^{\circ}(n \in \mathbb{Z})$ around any of its three main axes. This means that its opposite phases cannot be qualitatively distinguished, even if they are phases of the die that are numerically different. Hence, if an observer rotates the die $\mathrm{B} 180^{\circ}$ around one of its main axes, (s)he will not observe any difference between the initial and the final state of the die. On the contrary, if an observer rotates the die A around one of its main axes (excluding rotations of $360^{\circ}$ ), (s) he will observe phases of the die that are qualitatively different, in spite of the fact that (s)he is always observing the same die. This example suggests that phase transformations can produce "observable" 
effects, even if they do not modify the object as such. In particular, the six possible outcomes of a toss of a normal die are qualitatively different, in spite of the fact that these outcomes are just non-objective phases of the die.

To sum up the pre-ontology, we can say that there is no objective entity without an invariant identity ([34], p. 23), nor without different noninvariant phases. We shall now claim that this pre-ontology does not convey a complete ontology of physical objects. The reason for this is that the phase group of a given object-and therefore the corresponding objective invariants-remain for the moment unspecified. This problem was clearly stated by Nozick in the following terms: 'The notion of invariance under transformations cannot (without further supplementation) be a complete criterion of the objectivity of facts, for its application depends upon a selection of which transformations something is to be invariant under.' ([31], p. 79). In order to specify the phase group that defines the objective invariants of an object, we have to supplement the pre-ontology with an additional criterion. We shall now examine two alternative strategies for doing so.

Firstly, we could stress that the different phases of an object can be observed by performing spatiotemporal rotations and translations of the object. Hence, the group of phase transformations should be related to the kinematical symmetry group of the geometric background in which the object is embedded. Following this line of reasoning, we could try to adapt the strategy developed in Wigner's seminal 1939 paper [41] to the conceptual framework provided by the pre-ontology (see also Refs.[5, 28, 38]). ${ }^{7}$ More precisely, the space of phase orbits of the object should be a representation space for the kinematical group of the geometric background. In other terms, the phase orbits of an object embedded in a geometric background endowed with a particular kinematical group of symmetry (e.g. Galilei group, Poincaré group, etc.) should be generated by elements of a representation of the corresponding group. However, this proposal does not solve the problem of the indetermination of the phase group. In order to show this, we can argue as follows. We want to understand why the description of a physical system in terms of the canonical variables $(q, p)$ of a symplectic manifold

\footnotetext{
${ }^{7}$ Philosophical discussions of Wigner's description of "elementary particles" in terms of irreducible representations of the relevant kinematic group of symmetries can be found in Refs.[18, 19, 30, 35].
} 
$(P, \omega)$ is not satisfactory. In the terms provided by the geometric quantization formalism, we want to understand why it is necessary to extend the symplectic manifold $(P, \omega)$ to a $U(1)$-complex linear bundle $L \rightarrow P$ endowed with a connection and a compatible Hermitian metric. However, the information according to which the canonical variables $(q, p)$ of a symplectic manifold describe the embedding of the corresponding physical system in a spatiotemporal manifold is an additional piece of information that is not encoded in the symplectic manifold itself. In other terms, nothing in a symplectic manifold $(P, \omega)$ forces us to assume that the observables $f \in \mathcal{C}^{\infty}(P)$ describe a spatiotemporal dynamics. In particular, there exist physical systems, like for instance the inertio-gravitation field of general relativity, that by definition are not embedded in an external geometric stage. Indeed, the canonical variables of the ADM Hamiltonian formulation of general relativity describe (in a particular gauge) the temporal evolution of the Riemannian geometry of spatial hypersurfaces [3, 29]. This means that the Hamiltonian formulation of general relativity does not describe the dynamics of some canonical degrees of freedom in space, but rather the temporal evolution of the geometry of space itself. Since the final objective of this analysis is to construct a quantum ontology valid for any Hamiltonian system, we cannot presuppose that the corresponding observables can always be interpreted in terms of an embedding of the corresponding system in a geometric background. Hence, the only geometric setting that we shall presuppose is the symplectic manifold $(P, \omega)$ that describes the possible states of the classical system. An advocate of a Wignerian approach could still reply that in the case of general relativity and classical Yang-Mills theory the group of phase transformations could be identified with the group of general diffeomorphisms of the Lorentzian manifold $M$ and the gauge group (i.e. the group of vertical automorphisms of the corresponding $G$-principal fiber bundle over $M$ ) respectively. However, these groups are generated by the first-class constraints of the corresponding theories. This means that the corresponding gauge symmetries can be eliminated (at least in principle) by passing to the unconstrained reduced phase space description. By doing so, we obtain ordinary (i.e. unconstrained) classical Hamiltonian systems. The universal phase symmetry must explain why the classical description provided by this reduced phase space is overdetermined, even if the gauge group action has already been quotiened out. Since our objective is to con- 
struct an ontology of physical systems capable of explaining the necessity of passing from the $2 n$-dimensional classical description of an unconstrained Hamiltonian system to the $n$-dimensional quantum description, we cannot identify the phase group that induces this reduction with a gauge group. All in all, whereas gauge symmetries generated by $k$ first-class constraints explain the symplectic reduction from the $2(n+k)$ original degrees of freedom of a constrained system to the $2 n$ degrees of freedom of the reduced phase space, phase symmetries must explain the reduction from these $2 n$ classical degrees of freedom to the $n$ quantum degrees of freedom.

A second proposal for trying to bypass the indetermination of the phase group of an object is to claim, in the wake of Klein's Erlangen program, that the symmetry group is fixed by the free (or conventional) choice of what Debs and Redhead call an "invariance criterion" [20]. We could then argue, as for instance Cassirer does, that the freedom in the choice of the group of symmetry transformations is a consequence of the fact that the objective invariants, far from being immediately given, result from an active determination of physical objectivity. ${ }^{8}$ This means that what counts as an objective invariant is not defined once for all, but rather depends on the kind of properties that the subject considers relevant in a given research framework [19]. ${ }^{9}$ However, this proposal does not permit us to overcome the indetermination of the phase group for the following reason. We want to use the action of the phase group to explain the "projection" from the $2 n$ classical canonical variables to the $n$ quantum observables that define quantum states. We know from quantum mechanics that this "projection" depends on the quantum system. For instance, a quantum system can be localized either in $q$ or in $p$, i.e. be an eigenstate either of $\hat{q}$ or $\hat{p}$. If we assumed that the phase group is fixed by the free election of an invariance criterion, then

\footnotetext{
${ }^{8}$ Taking our departure from a fact given in intuition, there are altogether different directions in which we may proceed and determine the fact accordingly, i.e., according to the group of transformations to which we may refer. We enjoy complete freedom in the choice of these alternative groups. Different groups will yield different invariants and hence different geometrical properties.' ([16], p. 14; see also Ref.[17]).

${ }^{9}$ Weyl subscribes this solution in the following terms: 'We found that objectivity means invariance with respect to the group of automorphisms. Reality may not always give a clear answer to the question what the actual group of automorphisms is, and for the purpose of some investigations it may be quite useful to replace it by a wider group.' ([40], p. 132). In Ref.[26] Ladyman describes Weyl's notion of objectivity in the following terms: "Objects are picked out by individuating invariants with respect to the transformations relevant to the context."
} 
the difference between a state localized in $q$ and a state localized in $p$ would become purely conventional. Hence, the very physical difference between eigenstates of different quantum operators would disappear. As we know from quantum mechanics, it is the choice of a representation-i.e. the choice of a basis of the Hilbert space-that results from a free choice. On the contrary, the fact that a quantum state is an eigenstate of a certain complete set of commuting operators defines the physical state as such. Moreover, there is no reason for presupposing that the objective properties of different objects of the same kind (i.e. described by the same space of states) must be invariant with respect to the same phase group. In the conceptual framework provided by the pre-ontology, two objects can differ in two ways. On the one hand, their invariants with respect to the same phase group can be different. In quantum mechanical terms, two quantum states can differ by the fact that they are eigenstates of the same complete set of commuting operators with different eigenvalues. Secondly, the objective properties of two different objects can be invariant with respect to different phase groups. In quantum mechanical terms, two quantum states can differ by the fact that they are eigenstates of different complete sets of commuting operators. This means that phase symmetry cannot result from the "subjective" freedom in defining the kind of transformations and invariants that the observer will take into consideration, but rather from the intrinsic structure of the objects themselves.

The indetermination of the phase group justifies the necessity of extending the pre-ontology to what we shall call quantum ontology. The latter can be obtained by adding two postulates to the former, namely the phase postulate and the quantum postulate. Whereas the phase postulate specifies the phase group that defines the objective properties of a given object, the quantum postulate uses this specification to provide a positive characterization of the information about the object that is conveyed by its objective properties. We shall then argue that these two postulates are formally implemented by the two steps of the geometric quantization of a symplectic manifolds, namely the prequantization and the election of a polarization of pre-quantum states. 


\section{Phase postulate}

According to the considerations of Section II, the localization (or individualization) of physical states in gauge theories depends on the existence of two kinds of properties, namely the first-class constraints and the observables. While the first-class constraints $G_{a} \in \mathcal{C}^{\infty}(P)$ allow us to define the reduced phase space $\left(P_{\text {red }}, \omega_{\text {red }}\right)$ of possible physical states of the theory, the observables $f \in \mathcal{C}^{\infty}\left(P_{\text {red }}\right)$ allow us to individualize the different physical states in $P_{r e d}$. We could say that the constraints define the kind of physical states that the theory takes into consideration. On the other hand, the observables can be used to individualize the different physical states of the corresponding kind. For instance, the presence of first-class constraints in the Hamiltonian formulation of general relativity implies that the possible physical histories described by the theory do not represent 4-dimensional manifolds $M$ endowed with Lorentzian metrics $g_{a b}$, but rather equivalence classes $\left[\left(M, g_{a b}\right)\right]$ of Lorentzian manifolds under general diffeomorphisms of $M$. Far from being a mere epistemic requirement, these constraints encode the ontological commitment of the theory, that is to say the kind of its possible physical states. ${ }^{10}$ Indeed, the existence of constraints in general relativity implies that the kind of possible physical states described by the theory is such that spatiotemporal locations on $M$ have no physical significance [36].

The important point for the present discussion is that these two classes of properties - i.e. the first-class constraints and the observables - are differently treated in classical mechanics. On the one hand, the first-class constraints - i.e. the properties that define the kind of possible physical states - "strike" twice. Firstly, the constraints define a localization (or, equivalently, a restriction) to the constraint surface $\Sigma \subset P$. Secondly, the constraints induce gauge transformations that define a projection $\Sigma \rightarrow P_{\text {red }}$ to the orbit space of possible physical states (reduced phase space). We could then say that the localization to the constraint surface defined by a set of first-class constraints $\left\{G_{a}\right\}$ entails a projection to the orbit space defined by the action of the associated classical operators $\left\{v_{a}\right\} .{ }^{11}$ We could rephrase

\footnotetext{
${ }^{10} \mathrm{An}$ analysis of this ontological interpretation of gauge symmetries for the particular case of classical Yang-Mills theory can be found in Ref.[13].

${ }^{11}$ The fact that the localization to the constraint surface defined by a first-class constraint $G_{a}$ always entails a projection along the gauge orbits generated by the Hamiltonian vector field $v_{a}$ explains why each constraint removes two unphysical canonical variables.
} 
this by saying that the restriction to the subspace of states that satisfy the constraint equation $G_{a}(q, p)=0$ entails the impossibility of identifying different elements of a single gauge orbit generated by $v_{a}$ with different physical states. It is worth stressing that this "limitation" does not result from a supposed incompleteness of the theory, but rather from the fact that gauge equivalent states are just different coordinate representations of the same physical state.

On the other hand, the observables on the reduced phase space $f(q, p) \in$ $\mathcal{C}^{\infty}\left(P_{\text {red }}\right)$ (or, in general, on any unconstrained phase space) "strike" only once. ${ }^{12}$ Let's consider for instance the subspace of states that satisfy the property $p=p_{0}$. The important point is that the restriction to the surface $p=p_{0}$ does not entail a projection defined by the action of the classical operator $v_{p}=\frac{\partial}{\partial q}$ (which generates translations along the coordinate $q$ ). In other terms, the fact that the momentum $p$ of a physical system has the value $p_{0}$ does not imply that the transformations between the different values of $q$ are mere symmetry transformations. Hence, the restriction to the surface defined by a particular value of $p$ does not forbid us from identifying each value of the canonically conjugated coordinate $q$ with a different physical state. In fact, in classical mechanics it is necessary to fix the values of both $q$ and $p$ in order to individualize a physical state. We can thus conclude that in classical mechanics a localization defined by an observable does not entail a projection along the canonically conjugated observable.

To summarize, we can say that a classical observable only defines a localization, whereas a first-class constraint defines both a localization (to the constraint surface) and a projection (to the reduced phase space). The first postulate of the quantum ontology removes this difference between observables and first-class constraints:

Phase postulate: the transformations induced by an observable $f$ that defines an objective property of an object are phase transformations of the object.

In other terms, whereas first-class constraints induce gauge transformations, the transformations induced by the objective properties of an ob-

\footnotetext{
${ }^{12}$ For the sake of simplicity, we use the same letters $q$ and $p$ for denoting the canonical variables of both $P$ and $P_{\text {red }}$.
} 
ject should be understood as phase transformations. We are thus claiming that the correspondence between properties and symmetry transformations should be valid for both the properties that define the kind of possible physical states (first-class constraints) and the properties that individualize the different physical states of the same kind (observables). In other terms, a localization - be it defined by a first-class constraint or by an observablemust always entail a projection. Let's consider for instance a physical system characterized by the objective property $p=p_{0}$. According to the phase postulate, the transformations generated by the operator associated to $p$ (i.e. the translations along the coordinate $q$ ) must be interpreted as phase transformations of the corresponding object. Hence, we can say that the phase transformations induced by $p$ "phase out" the coordinate $q$, which means that the different values of $q$ are just "pure phase". In this way, the phase postulate explains why a quantum physical system cannot be described by $2 n$ sharp objective (or phase invariant) properties.

The phase postulate permit us to define the group of phase transformations of a given object in the following terms: the phase group of an object is composed of all the transformations induced by the objective properties of the object. Hence, the phase group is object-dependent: two objects defined by the values $\left\{f_{\alpha}^{1}, \ldots, f_{\rho}^{n}\right\}$ and $\left\{g_{\beta}^{1}, \ldots, g_{\varrho}^{n}\right\}$ of the different set of observables $\left\{f^{1}, \ldots, f^{n}\right\}$ and $\left\{g^{1}, \ldots, g^{n}\right\}$ respectively have different phase groups, namely the groups of phase transformations generated by the operators associated to these two different sets of observables.

The phase postulate implies that we cannot identify each point of a symplectic manifold with a different physical state, even if the system has no constraints. We could say that points in phase space provide an overdetermined description of physical systems, since they do not only define the objective properties of the system (e.g. $p_{0}$ ) but also select one particular representant (e.g. $q_{0}$ ) of the orbit generated by the action of the operator (e.g. $v_{p}$ ) associated to the objective property in question. ${ }^{13}$ We can thus conclude that phase space is not an adequate geometric arena for defining physical systems that satisfy the phase postulate. It is worth stressing that this conceptual justification of the reduction in the number of variables that

\footnotetext{
${ }^{13}$ Analogously, points in the original phase space of a constrained Hamiltonian system provide an overdetermined description of physical states, since they fix the values of $2 k$ non-physical canonical variables (where $k$ is the number of first-class constraints).
} 
are necessary to completely decribe a physical system does not presuppose any kind of epistemic restriction to the amount of information an observer can have about an object.

\section{Quantum postulate}

The phase postulate states that the objective properties of a physical system induce, by means of the action generated by theirs associated operators, the phase transformations between its non-objective phases. We shall now analyze the relationship between the possible numerical values of the objective properties and the phase transformations generated by theirs associated operators. In other terms, we want to understand the relationship between the two fundamental roles played by physical observables in mechanics, namely (1) to assign numerical values to physical states, and (2) to define operators that act on states. To do so, we shall begin by remarking a tautological fact, namely that by definition a velocity $\dot{q}$ conveys information about the instantaneous rapidity of the transformation of $q$ in time. Analogously, we could expect the particular value of a momentum $p$ to convey information about the transformations that are naturally associated to it, namely the canonical transformations of $q$ generated by the classical operator $v_{p}$. However, in classical mechanics the different values of an observable $f \in \mathcal{C}^{\infty}(P)$ do not convey any information about the transformations generated by the classical operator $v_{f} \in T P$ associated to $f$. For

instance, the transformations of $q$ generated by $v_{p}=\frac{\partial}{\partial q}$ do not depend on the numerical value of $p$. Indeed, $p$ and $p^{\prime}=p+k$ (with $k \in \mathbb{R}$ ) define the same infinitesimal transformation of $q$ :

$$
\mathcal{L}_{i e_{p+k}} q=\{q, p+k\}=\{q, p\}=\mathcal{L} i e_{v_{p}} q
$$

This is a consequence of the fact that the surjective Lie algebra homomorphism (1) between observables $f \in \mathcal{C}^{\infty}(P)$ and classical operators $v_{f} \in \mathcal{H}_{P}$ is not injective (or faithful), being its kernel the set of constant 
functions ${ }^{14}$ :

$$
f(q, p)=k \in \mathbb{R} \mapsto v_{k}=\frac{\partial k}{\partial p} \frac{\partial}{\partial q}-\frac{\partial k}{\partial q} \frac{\partial}{\partial p}=0 .
$$

We can thus conclude that the non-injectivity of the Lie algebra homomorphism (1) between the Poisson algebra of observables $\mathcal{C}^{\infty}(P)$ and the Lie algebra $\mathcal{H}_{P}$ of classical operators implies that the canonical transformations generated by $v_{p}$ do not depend on the particular value of $p$. It is worth noting that this fact can also be considered a consequence of the commutativity of the classical operators $v_{p}$ and $v_{q}$. Indeed, since the translation from $p$ to $p^{\prime}=p+k$ is generated by the classical operator $v_{q}=-\frac{\partial}{\partial p}$ associated to $q$, the variation of $v_{p}$ under a transformation of $p$ is given by the Lie derivative of $v_{p}$ along $v_{q}$. In other terms, the Lie derivative $\mathcal{L} i e_{v_{q}} v_{p} \doteq\left[v_{p}, v_{q}\right]$ measures how the classical operator $v_{p}$ changes under a transformation of $p$ (generated by definition by $v_{q}$ ). The important result is that the non-injectivity of the Lie algebra homomorphism (1) implies that this Lie derivative is zero:

$$
\mathcal{L}_{i e_{q}} v_{p}=\left[v_{p}, v_{q}\right]=v_{\{q, p\}}=v_{1}=0 .
$$

Therefore, the commutativity of the classical operators associated to canonically conjugated variables implies that the numerical values of the latter (e.g. $p$ ) do not faithfully quantify the transformations generated by theirs associated classical operators (e.g. $v_{p}$ ). In order to bypass this flaw of the Lie algebra homomorphism (1) between observables and classical operators, we shall introduce the second postulate of the quantum ontology:

Quantum postulate: the numerical value of an objective property of an object must faithfully quantify the phase transformations generated by its associated operator.

The previous arguments show that the implementation of this postulate requires to force the injectivity of the Lie algebra homomorphism between

\footnotetext{
${ }^{14}$ This property of the application between observables $f \in \mathcal{C}^{\infty}(P)$ and Hamiltonian vector fields $v_{f} \in \mathcal{H}_{P}$ can be summed up by saying that the short sequence

$$
0 \rightarrow \mathbb{R} \stackrel{i}{\rightarrow} \mathcal{C}^{\infty}(P) \stackrel{\pi}{\rightarrow} \mathcal{H}_{P} \rightarrow 0
$$

is exact. In other words, the image of the injection $i$ - the constant functions $f=k$ in $\mathcal{C}^{\infty}(P)$ - is the kernel of the projection $\pi$. This means that $\mathcal{H}_{P}=\mathcal{C}^{\infty}(P) / \mathbb{R}$.
} 
observables and operators. Equivalently, in order to implement a faithful correspondence between the two roles played by physical observablesnamely, to assign numerical values to physical states and to induce canonical transformations - , it is necessary to force the non-commutativity of the operators associated to complementary pairs of observables. In this way, the quantum postulate permits us to understand the conceptual scope of the formal analogy between Poisson brackets of classical observables and commutators of quantum operators proposed by Dirac. The essential difference between classical and quantum mechanics does not rely on the existence of a map between observables $f$ and operators $O_{f}$ such that $\left[O_{f}, O_{g}\right]=O_{\{f, g\}}$ (condition which is already satisfied by the Lie algebra homomorphism (5) between observables $f \in \mathcal{C}^{\infty}(P)$ and classical operators $v_{f} \in T P$; see expression (6)), but rather on the requirement according to which such a map must be injective. This last requirement amounts to implement the Dirac quantization condition according to which quantum operators must verify

$O_{1}=\hat{1}$, where $\hat{1}$ is the identity operator.

A satisfactory implementation of the two postulates of the quantum ontology would permit us to define an objective property of an object as a quantity that faithfully (quantum postulate) quantifies the phase transformations of the object generated by its associated operator (phase postulate). In section VII, we shall argue that geometric quantization implements these two independent postulates of the quantum ontology in two different stages, namely the prequantization of a symplectic manifold and the election of a polarization. As we shall see, whereas the implementation of the quantum postulate requires to pass from classical operators to quantum operators, the implementation of the phase postulate requires to pass from classical states to quantum states. Before showing this, we shall further analyze the notion of objective properties that results from these postulates.

\section{Momenta vs. velocities}

It is worth stressing that the notion of objective properties that results from the phase postulate and the quantum postulate differs from the usual interpretation of physical observables. According to the standard comprehension of Hamiltonian mechanics, a momentum $p$ is just the canonical version of the velocity $\dot{q}(t)$. This means that $p$ indirectly measures, via its relation to $\dot{q}(t)$, the instantaneous rapidity of a physical motion along the 
coordinate $q$ parameterized by a temporal parameter $t$. The transformation from the dependent variables $q$ and $\dot{q}(t)$ to the independent canonical variables $q$ and $p$, i.e. the introduction of the auxiliary variables $p$, seems to be a mere trick to pass from the $n$ second-order Euler-Lagrange equations to the $2 n$ first-order Hamilton equations. However, the relation between $p$ and $\dot{q}(t)$ depends on the presupposition of a temporal structure, that is to say of a temporal variable $t$ and a conjugated Hamiltonian $h$. A temporal structure can be introduced in two different ways. For subsystems of the universe, the existence of a temporal structure results from the presupposition of an external temporal parameter $t$ and the definition of a Hamiltonian function $h$ on the phase space of the subsystem in question. In a cosmological framework, where by definition there are no external variables, a temporal structure can be defined by selecting a suitable degree of freedom to play the role of an internal physical clock. These kinds of systems are characterized by the presence of the so-called Hamiltonian constraint $H=0$. The election of an internal temporal structure amounts to fix the gauge [15]. The important point in the present context is that a temporal structure (either external or internal) is an additional layer of structure that is not encoded in the symplectic structure of phase space. In order to stress this fact, we shall establish a difference between a symplectic system $(P, \omega)$ and a Hamiltonian system $(P, \omega, t, h)$, that is a symplectic system endowed with a temporal structure.

The point that we want to stress here is that the notion of objective properties conveyed by the phase postulate and the quantum postulate, far from presupposing the existence of a temporal structure, only relies on the symplectic structure of phase space. As we explained in Section II, the classical operators $v_{f} \in \mathcal{H}_{P}$ defined by the Lie algebra homomorphism (1) act on physical states by means of symplectic diffeomorphisms (or canonical transformations) of $(P, \omega)$. In particular, the momentum $p$ induces canonical transformations of the coordinate $q$ through the action of the classical operator $v_{p}=\frac{\partial}{\partial q}$. If the symplectic system is endowed with a temporal structure $(t, h)$, then we can legitimately claim that the momentum $p$, being related to the velocity $\dot{q}(t)$ through Hamilton's equation $\dot{q}(t)=\frac{\partial h}{\partial p}$, indirectly measures the rapidity of the transformations of $q$ in time. Let's suppose, for instance, a standard Hamiltonian system with Hamiltonian function $h(q, p)=\frac{p^{2}}{2 m}+V(q)$. As we argue in Section V, the canonical 
transformations of $q$ induced by $p$ are not faithfully quantified by the numerical values of $p$. On the contrary, the transformations of $q$ in time are faithfully quantified by the value of the momentum $p$, since $p=m \dot{q}(t)$. However, this is just a consequence of that fact that the Hamiltonian function $h$ is quadratic in $p$. Indeed, Hamilton's equation $\frac{d q}{d t}=\frac{\partial h}{\partial p}$ means that the differential relationship between the transformations of $q$ (induced by $p$ ) and the transformations of $t$ (induced by $h$ ) depends on the functional relation between the generators of the corresponding transformations, namely $p$ and $h$ respectively. For instance, if the Hamiltonian $h$ were linear in $p$, then there would be no relation between $\dot{q}$ and $p$. The important point in the present context is that the canonical transformations of $q$ generated by $p$ can be related to the transformations of $q$ in time (i.e. to a temporal physical motion of the system) only through the mediation of the Hamiltonian $h$. If the system is not endowed with a temporal structure, then we cannot pass from canonical transformations to temporal physical motions. We shall then maintain that a physical interpretation of $p$ valid for any symplectic system (be it endowed with a temporal structure or not) must stem from the intrinsic definition of $p$, that is from the definition according to which $p$ is an observable that induces canonical transformations of $q$ through the action of its associated operator. Hence, it is necessary to construct a satisfactory physical interpretation of the canonical transformations induced by $p$. The phase postulate carries out this task. According to this postulate, the canonical transformations induced by an objective property of an object must be interpreted as phase transformations between the different non-objective phases of the object. However, the interpretation of canonical transformations as phase transformations would not be entirely satisfactory if the numerical values of the objective property in question were not related to the induced phase transformations. This problem is solved by means of the quantum postulate. Indeed, if the application between observables and operators were injective, then the numerical value of an objective property could be interpreted as a faithful numerical characterization of the way in which the object transforms under the corresponding phase transformations.

The implementation of the quantum postulate and the phase postulate would allow us to maintain that a momentum $p$, far from being intrinsically related to a velocity $\dot{q}$, must be understood as a quantity that faithfully quantifies (quantum postulate) the phase transformations of $q$ generated by 
the operator associated to $p$ (phase postulate). This means that the different values of the coordinate $q$ of an object characterized by a sharp value of $p$ do not describe the different instantaneous positions of a moving pointlike object, but rather the different "positional" phases of an intrinsically delocalized object. ${ }^{15}$ The important point is that this interpretation of the canonical transformations induced by an objective property does not depend on the possibility of realizing these transformations as effective motions in time. In particular, the objective properties of a symplectic system that is not endowed with a temporal structure, far from describing the temporal motion of the system, provide, by inducing the corresponding phase orbits, a faithful description of its atemporal multifaceted structure. By adopting the terminology introduced by Weyl in Ref.[39], we shall say that the transformations of an object induced by its objective properties, far from being actual displacements of the object in time, are virtual transformations of the object. If the system is endowed with a temporal structure, then these virtual transformations can be turned into actual displacements in time by measn of the corresponding Hamilton equation.

It is worth remarking that the rapidity of the motion in time of different physical systems can be quantified and mutually compared thanks to the existence of the standard provided by the common flow of time. In other terms, the difference between two velocities $\dot{q}_{1}(t)$ and $\dot{q}_{1}(t)$ reflects the fact that the corresponding systems traverse different distances in the same (infinitesimal) period of time. If we want to construct a satisfactory interpretation of an observable like a momentum $p$ by making abstraction of the existence of the standard provided by the flow of time, then we have to determine which is the common standard that permits us to distinguish

\footnotetext{
${ }^{15}$ In Ref.[11] (section III) we analyzed the compatibility between such an "atemporal" description of a free particle and the standard description in terms of the temporal evolution of the corresponding degrees of freedom. Briefly, the energy $E=\frac{p_{0}}{2 m}$ of a free particle characterized by the objective property $p=p_{0}$ is also an objective property of the particle. Hence, the phase postulate implies that the transformations induced by the Hamiltonian $h=\frac{p}{2 m}$ (i.e. the transformations of $t$ ) are mere phase transformations of the particle. In other terms, the fact that the Hamiltonian $h$ is an objective property of the particle implies that the temporal evolution of the system is just a phase transformation. It follows that the different positions $q(t)$, far from being instantaneous objective properties of a particle that is objectively moving in time, are just different phases of the system parameterized by $t$. Hence, the phase postulate implies that a free "particle", far from being a point-like object moving in time, should be understood as a delocalized object endowed with different temporal phases.
} 
two different values of $p$. The answer to this problem is provided in Ref.[12]. By using the so-called momentum map formalism, we showed that the injectivity of the map between observables and operators would permit us to define physical observables as quantities that faithfully quantify the representation of abstract operators belonging to the corresponding Lie algebra $\mathfrak{g}$ as operators acting on the space of states. In particular, different values of the observable $p$, far from characterizing the transformations of $q$ with respect to the common flow of time, would quantify the different possible representations of the same abstract operator $\xi_{q} \in \mathfrak{g}{ }^{16}$

\section{Geometric quantization in the light of the quantum ontology}

The phase postulate and the quantum postulate cannot be implemented in the framework of classical mechanics. Firstly, the representation of physical systems by means of points in phase space does not satisfy the phase postulate. In fact, this postulate implies that classical states are overdetermined, since they do not only define the objective properties of physical systems (e.g. $p_{0}$ ) but also select one particular value (e.g. $q_{0}$ ) of the phase orbit generated by the operator (e.g. $v_{p}$ ) associated to the objective property. This means that non-objective properties are wrongly considered objective in classical mechanics. Secondly, the non-injective Lie algebra homomorphism (1) between observables $f \in \mathcal{C}^{\infty}(P)$ and classical operators $v_{f} \in \mathcal{H}_{P}$ does not satisfy the quantum postulate. We shall now argue that

\footnotetext{
${ }^{16}$ The main geometric ingredients of this construction are the following. The symplectic action $\Phi: G \times P \rightarrow P$ of a Lie group $G$ of Lie algebra $\mathfrak{g}$ on a manifold $P$ defines a map $\iota: \mathfrak{g} \rightarrow T P$ between Lie algebra elements $\xi \in \mathfrak{g}$ and the so-called fundamental vector fields $v_{\xi}$ on $P$ by means of the expression $v_{\xi}(x)=\left.\frac{d}{d \lambda}(\exp (-\lambda \xi) \cdot x)\right|_{\lambda=0}($ for $x \in P)$. In Ref.[12], we introduced the following terminology: the fundamental vector field $v_{\xi} \in T P$ evaluated at a point $x \in P$ is said to be the representation of the abstract operator $\xi \in \mathfrak{g}$ on the particular state $x$. A symplectic $G$-action is said to be Hamiltonian if there exists a map $\tilde{\mu}: \mathfrak{g} \rightarrow \mathcal{C}^{\infty}(P)$ (called co-momentum map) such that $\tau \circ \tilde{\mu}=\iota$, where $\tau: \mathcal{C}^{\infty}(P) \rightarrow \mathcal{H}_{P}$. This means that the representation $\iota$ of abstract operators in $\mathfrak{g}$ as fundamental vector fields in $T P$ is factorized through the physical observables in $\mathcal{C}^{\infty}(P)$ :

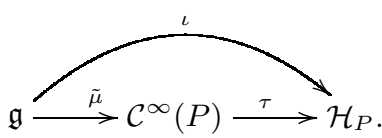

If the maps $\tilde{\mu}$ and $\tau$ were equivariant and injective respectively, then we could state that observables in $\mathcal{C}^{\infty}(P)$ faithfully quantify the representation on $P$ of abstract operators in $\mathfrak{g}$. However, this is not the case in classical mechanics (see Ref.[12] for details).
} 
the canonical quantization of a symplectic manifold $(P, \omega)$ amounts to implement these two postulates. Our arguments rely on the most rigorous mathematical constructive formalization of canonical quantization, namely geometric quantization $[7,33,37,42] .^{17}$

According to the canonical quantization procedure, the self-adjoint quantum operators $\hat{v}_{f}$ associated to observables $f \in \mathcal{C}^{\infty}(P)$ must satisfy the following conditions:

$\left.Q_{1}\right)$ If $f=k \in \mathbb{R}$, then $\hat{v}_{f=k}=k I$, where $I$ is the identity operator.

$\left.Q_{2}\right)$ If $\{f, g\}=h$, then $\left[\hat{v}_{f}, \hat{v}_{g}\right]=-i \hbar \hat{v}_{h}$.

$\left.Q_{3}\right)$ If $\left\{f_{i}\right\}$ is a complete set of classical observables, then the set $\left\{\hat{v}_{f_{i}}\right\}$ must act irreducibly on the Hilbert space of quantum states. ${ }^{18}$

Condition $Q_{2}$ states that the application from observables to operators must be a Lie algebra homomorphism. However, expression (6) shows that this condition is already satisfied by the classical application (1) between observables $f \in \mathcal{C}^{\infty}(P)$ and classical operators $v_{f} \in \mathcal{H}_{P}$. Hence, far from being introduced when passing to the quantum description, the existence of an application between Poisson brackets of observables and commutators of operators is an essential feature of classical mechanics. This means that strictly speaking canonical quantization only relies on two fundamental conditions, namely $Q_{1}$ and $Q_{3}$.

Condition $Q_{1}$ states that the Lie algebra homomorphism between observables and operators must be injective. In the framework of geometric quantization, this condition is implemented by means of the so-called prequantization formalism. This formalism shows that an operator algebra isomorphic to the Poisson algebra $\mathcal{C}^{\infty}(P)$ of classical observables can be defined by extending classical operators $v_{f}$ to quantum operators $\hat{v}_{f}$. To do so, it is necessary to define a $U(1)$-principal fiber bundle $L \stackrel{\vartheta}{\rightarrow} P$ over the phase space $(P, \omega)$ endowed with a connection $\tilde{\theta}$ of curvature defined

\footnotetext{
${ }^{17}$ Short introductions to geometric quantization can also be found in Refs.[1, 27].

${ }^{18} \mathrm{~A}$ set of smooth functions $\left\{f_{i}\right\}$ on $P$ is said to be a complete set of classical observables if every other function $g$ that satisfies $\left\{f_{i}, g\right\}=0$ for all $f_{i}$ is necessarily constant. This implies that the complete set $\left\{f_{i}\right\}$ locally separates points in $P$. In other terms, the values $\left\{f_{i}(x)\right\}$ provided by a complete set of classical observables suffice to individualize the state $x \in P$. In turn, a set of self-adjoint operators $\left\{\hat{v}_{f_{i}}\right\}$ acting on a Hilbert space $\mathbf{H}$ is said to be a complete set of operators if every other operator $\hat{v}_{g}$ that commutes with all of them is a multiple of the identity.
} 
by the symplectic form $\omega \cdot{ }^{19}$ Thanks to the existence of the new "vertical" dimensions defined by the fibers $\vartheta^{-1}(x)$ (with $x \in P$ ), it is possible to add vertical components $\zeta_{f}$ tangent to the fibers to the classical operators $v_{f} \in T P$. Moreover, this can be done in such a way that the extended vector fields $\hat{v}_{f}=v_{f}^{h}+\zeta_{f}$ (where $v_{f}^{h}$ is the horizontal lift of $v_{f}$ defined by the connection $\tilde{\theta}$ ) satisfy commutation relations isomorphic to the Poisson algebra of observables. In this way, the prequantization formalism shows that the quantum operators $\hat{v}_{f}$ can be obtained by means of a suitable extension of the classical operators $v_{f}$.

The quantum operators $\hat{v}_{f}$ act by construction on functions on $L$. This action can be used to define an induced action on the so-called pre-quantum states, that is on the sections of the associated fiber bundle $\tilde{L}=L \times_{U(1)} \mathbb{C} \stackrel{\pi}{\rightarrow}$ $P$. Let's denote $\mathcal{H}(P, L)$ the space of these sections. More precisely, it can be shown that the quantum operators $\hat{v}_{f}$ act on sections $\psi: P \rightarrow \tilde{L}$ by means of the following expression ([7], Proposition 2.3.16):

$$
\hat{v}_{f}(\psi)=-i \hbar \nabla_{v_{f}} \psi+f \psi,
$$

where $\nabla_{v_{f}}=v_{f}+\frac{i}{\hbar} \tilde{\theta}\left(v_{f}\right)$.

Let's consider for instance the cotangent bundle $P=T^{*} Q$. Since the curvature of the connection $\tilde{\theta}$ is given by the symplectic form $\omega$, the local connection form coincides with the canonical 1-form $\theta$ of the symplectic manifold $(P, \omega)$. By choosing the local connection form $\theta=-p d q$, the quantum operators associated to $q$ and $p$ take the form:

$$
\hat{v}_{q}=i \hbar \frac{\partial}{\partial p}+q, \quad \hat{v}_{p}=-i \hbar \frac{\partial}{\partial q} .
$$

By endowing the associated fiber bundle $\tilde{L} \rightarrow P$ with an Hermitian inner product compatible with the connection $\nabla$, expression (9) defines Hermitian operators with respect to the inner product

$$
\langle\psi, \varphi\rangle=\int_{P}\langle\psi(x), \varphi(x)\rangle \frac{\omega^{n}}{n !}
$$

where $\psi$ and $\varphi$ are sections of $\tilde{L}$.

\footnotetext{
${ }^{19}$ It is possible to show that this geometric construction exists if an only if $\omega$ satisfies the so-called integrality condition, i.e. if an only if $(2 \pi \hbar)^{-1} \omega$ defines an integral cohomology class in $H^{2}(P, \mathbb{Z})$. It is worth remarking that the discrete character of some quantum operators' spectra can be derived from this topological condition (see Refs.[7, 33, 37, 42] for details).
} 
The extension of classical operators $v_{f}$ to quantum operators $\hat{v}_{f}$ does not suffice to reobtain quantum mechanics. In fact, since pre-quantum states $\psi: P \rightarrow \tilde{L}$ can be localized in both $q$ and $p$, they violate Heisenberg uncertainty principle. The fact that the Hilbert space $\mathcal{H}(P, L)$ of pre-quantum states is so large implies that the quantum operators associated to a complete set of classical observables do not act irreducibly on the Hilbert space of pre-quantum states. Hence, the Hilbert space of pre-quantum states does not satisfy condition $Q_{3} \cdot{ }^{20}$ In the framework of geometric quantization, this problem can be solved-i.e. condition $Q_{3}$ can be satisfied-by introducing an additional structure, namely a polarization. Roughly speaking, the election of a polarization "cuts in half" the Hilbert space $\mathcal{H}(P, L)$ of pre-quantum states. A polarization $\mathcal{P}$ of a symplectic manifold $(P, \omega)$ is a foliation of $P$ by Lagrangian (i.e. maximally isotropic) submanifolds. A Lagrangian submanifold of a $2 n$-dimensional symplectic manifold $(P, \omega)$ is a $n$-dimensional submanifold $K \subset P$ such that $\omega$ vanishes on $T_{x} K \times T_{x} K$. A canonical example of a Lagrangian submanifold is the configuration space $Q$ of the cotangent bundle $P=T^{*} Q$. The identity $i_{v_{g}} i_{v_{f}} \omega=\{f, g\}$ implies that the observables associated to the Hamiltonian vector fields that define a polarization form a complete set of commuting observables. A section $\psi: P \rightarrow \tilde{L}$ is said to be polarized with respect to the polarization $\mathcal{P}$ if it is covariantly constant along $\mathcal{P}$, i.e. if it satisfies

$$
\nabla_{\mathcal{P}} \psi=0 .
$$

Let's consider for instance the so-called vertical polarization of the cotangent bundle $P=T^{*} Q$, i.e. the polarization spanned by the vector field $\frac{\partial}{\partial p}$. If we choose the local connection form $\theta=-p d q$, then the polarized sections are the functions on $T^{*} Q$ that satisfy $\frac{\partial \psi}{\partial p}=0$, that is the functions that are constant along the fibers of the cotangent bundle $T^{*} Q$. The resulting polarized states only depend on the coordinate $q$ of the configuration space $Q$ (Schrödinger representation). Analogously, the momentum representation can be obtained by using the polarization spanned by the vector field $\frac{\partial}{\partial q}$. These trivial examples show that the election of a particular polarization

\footnotetext{
${ }^{20}$ Let's consider for instance the subset of states of the form $\psi(q)$. The states $\hat{v}_{q} \psi(q)=$ $q \psi(q)$ and $\hat{v}_{p} \psi(q)=-i \hbar \frac{\partial \psi(q)}{\partial q}$ that result from applying the operators (10) to this subset of states also depend only on $q$. Hence, the states $\psi(q)$ define a proper subspace of the set of pre-quantum states that is invariant under the action of the quantum operators $\hat{v}_{q}$ and $\hat{v}_{p}$.
} 
amounts to choosing a representation of the corresponding quantum theory.

We shall now argue that this factorization of canonical quantization in two independent stages can be understood as a formal implementation of the two postulates of the quantum ontology. To do so, it is necessary to further specify the relationship between the conceptual framework provided by the quantum ontology on the one hand and the quantum formalism on the other. This can be done by identifying the objective properties of a quantum system with the eigenvalues (or quantum numbers) that define the corresponding quantum vector. In other terms, we assume the validity of the so-called eigenvalue-eigenstate link [8, 22]. More precisely, we assume that the numerical value $f_{0}$ of the observable $f$ is an objective property of the object represented by the pure state $\psi$ if and only if $\psi$ is an eigenstate of $\hat{v}_{f}$ with eigenvalue $f_{0}$, i.e. if and only if $\hat{v}_{f} \psi=f_{0} \psi$. In what follows, $\left\{f^{1}, \ldots, f^{n}\right\}$ is a complete set of commuting observables and $f_{\alpha}^{j}$ denotes the $\alpha$ eigenvalue of $\hat{v}_{f^{j}}$ (which will be written, for the sake of simplicity, as $\hat{v}_{j}$ ). The eigenvalue-eigenstate link allows us to introduce the main ontological claim of the proposed interpretative framework, namely that the physical referent of a pure state $\psi=\left|f_{\alpha}^{1}, \ldots, f_{\rho}^{n}\right\rangle$ is a single "multifaceted" structure defined by the invariant objective properties $\left\{f_{\alpha}^{1}, \ldots, f_{\rho}^{n}\right\}$ that faithfully quantify the virtual phase transformations of the object generated by the self-adjoint operators $\left\{\hat{v}_{1}, \ldots, \hat{v}_{n}\right\}$.

As we argued in Section V, the non-injectivity of the Lie algebra homomorphism $f \mapsto v_{f}$ between observables and classical operators implies that the numerical values obtained by evaluating the observables $f$ on physical states $x \in P$ do not faithfully quantify the transformations generated by theirs associated classical operators $v_{f}$. As we have just explained, the prequantization formalism extends classical operators $v_{f}$ to quantum operators $\hat{v}_{f}$ in such a way that the application $f \mapsto \hat{v}_{f}$ is injective. Hence, we could expect the transformations generated by the quantum operators $\hat{v}_{f}$ to be faithfully quantified by the numerical values of the corresponding observables. On the other hand, the polarization of prequantum states implemented by means of equation (11) implies that the resulting quantum states cannot depend on the variables that span the corresponding Lagrangian submanifold of phase space. As we explained before, these variables form a complete set of $n$ commuting observables (e.g. $\left\{q^{1}, \ldots, q^{n}\right\}$ ). Hence, the quantum states can only depend on the remaining $n$ commuting observ- 
ables (e.g. $\left.\left\{p_{1}, \ldots, p_{n}\right\}\right) \cdot{ }^{21}$ Since, the canonical transformations induced by an observable like $p$ transform the canonically conjugated variable $q$, we can expect a polarized state characterized by a given value $p_{0}$ of $p$ to be invariant under the transformations generated by the quantum operator $\hat{v}_{p}$. Indeed, a polarized state characterized a given value $p_{0}$ of $p$ cannot also depend on $q$. Hence, a transformation of $q$ cannot modify the state $\left|\ldots, p_{0}, \ldots\right\rangle$ as such. In the terms provided by the quantum ontology, we expect the transformations generated by $\hat{v}_{p}$ to be virtual phase transformations of the object represented by the state $\left|\ldots, p_{0}, \ldots\right\rangle$. This means that we expect quantum states to satisfy the phase postulate. All in all, the implementation of both the quantum postulate and the phase postulate should guarantee that each eigenvalue $f_{\alpha}^{j}$ quantifies faithfully how the corresponding Lie algebra element $T^{j} \in \mathfrak{g}$ is represented as an operator $\hat{v}_{j}$ that generates virtual phase transformations of the state $\left|\ldots, f_{\alpha}^{j}, \ldots\right\rangle$. Let $g=e^{i T^{j} \theta} \in G$ be the one-parameter abstract subgroup of $G$ (obtained by means of the exponential map exp $: \mathfrak{g} \rightarrow G$ ) whose tangent vector at the identity is equal to $T^{j}$. Let's consider now a quantum state $\left|\ldots, f_{\alpha}^{j}, \ldots\right\rangle$ characterized by the objective property $f^{j}=f_{\alpha}^{j}$. The abstract transformations $g=e^{i T^{j} \theta} \in G$ are represented as effective transformations of the state $\left|\ldots, f_{\alpha}^{j}, \ldots\right\rangle$ by means of the following expression:

$$
e^{i \hat{v}_{j} \theta}\left|\ldots, f_{\alpha}^{j}, \ldots\right\rangle=e^{i f_{\alpha}^{j} \theta}\left|\ldots, f_{\alpha}^{j}, \ldots\right\rangle
$$

This fundamental expression encompasses the two essential features of quantum mechanics implemented by the quantum postulate (i.e. by the prequantization formalism) and the phase postulate (i.e. by the election of a polarization). Firstly, the eigenvalue $f_{\alpha}^{j}$ quantifies faithfully how the abstract group elements $g=e^{i T^{j} \theta}$ are represented as effective transformations of the state. This means that two different eigenvalues $f_{\alpha}^{j}$ and $f_{\alpha^{\prime}}^{j}$ define different realizations $e^{i f_{\alpha}^{j} \theta}$ and $e^{i f_{\alpha^{\prime}}^{j} \theta}$ of the same abstract transformations $e^{i T^{j} \theta}$. In other terms, each eigenvalue $f_{\alpha}^{j}$ characterizes a particular representation of the group action defined by the one-parameter subgroup $e^{i T^{j} \theta}$

\footnotetext{
${ }^{21}$ This point has been clearly stated by Guillemin and Sternberg in the following terms: "The Heisenberg uncertainty principle says that it is impossible to determine simultaneously the position and momentum of a quantum-mechanical particle. This can be rephrased as follows: the smallest subsets of classical phase space in which the presence of a quantum-mechanical particle can be detected are its Lagrangian submanifolds. For this reason it makes sense to regard the Lagrangian submanifolds of phase space as being its true 'points'." [24], p. 515.
} 
of $G$. In this way, we could say that the quantum postulate guarantees that "all quantum numbers [...] are indices characterizing representations of groups." ([39], p. xxi). Expression (12) also encompasses the fact that the transformations of the state $\left|\ldots, f_{\alpha}^{j}, \ldots\right\rangle$ generated by the quantum operator $\hat{v}_{j}$ multiply the state by the phase $e^{i f_{\alpha}^{j} \theta}$, which means that they do not modify the state as such. This is the formal translation of the phase postulate, that is to say of the claim according to which the transformations induced by an objective property of an object (i.e. generated by the operator associated to one of the eigenvalues of the corresponding state) are virtual phase transformations of the object.

Let's consider, for instance, two quantum vectors, $\left|\frac{1}{2}, \frac{1}{2}\right\rangle$ and $|1,1\rangle$, such that $\hat{L}_{z}\left|\frac{1}{2}, \frac{1}{2}\right\rangle=\frac{\hbar}{2}\left|\frac{1}{2}, \frac{1}{2}\right\rangle$ and $\hat{L}_{z}|1,1\rangle=\hbar|1,1\rangle$ (where we have changed the notation for the quantum operators from $\hat{v}_{L_{z}}$ to the standard notation $\hat{L}_{z}$ ). According to the quantum postulate, the different eigenvalues of $\hat{L}_{z}$, being objective properties of the corresponding objects, must faithfully quantify the transformations generated by $\hat{L}_{z}$. More precisely, we expect the different eigenvalues of $\hat{L}_{z}$ to specify how the same abstract rotation around the vertical axis is differently represented as transformations of the corresponding quantum objects. Let's consider for example an abstract rotation given by $\theta_{z}=2 \pi$. While the quantum vector $|1,1\rangle$ does not change under such an abstract rotation:

$$
e^{i \hat{L}_{z} 2 \pi / \hbar}|1,1\rangle=e^{i 2 \pi}|1,1\rangle=|1,1\rangle,
$$

the quantum vector $\left|\frac{1}{2}, \frac{1}{2}\right\rangle$ "changes" in a sign:

$$
e^{i \hat{L}_{z} 2 \pi / \hbar}\left|\frac{1}{2}, \frac{1}{2}\right\rangle=e^{i \pi}\left|\frac{1}{2}, \frac{1}{2}\right\rangle=-\left|\frac{1}{2}, \frac{1}{2}\right\rangle .
$$

In this way, the different eigenvalues of $\hat{L}_{z}$ specify how the two quantum objects differently transform under the same abstract rotation around the $z$ axis. One could argue that strictly speaking the quantum vector $\left|\frac{1}{2}, \frac{1}{2}\right\rangle$ as such does not change under an abstract rotation given by $\theta_{z}=2 \pi$, since it only "changes" in the non-physical phase $e^{i \pi}$. Indeed, quantum vectors are defined up to a phase factor $e^{i \theta}$. However, far from being an objection, this simply means that quantum vectors also satisfy the phase postulate. In other terms, the transformations generated by the operators $\left\{\hat{v}_{1}, \ldots, \hat{v}_{n}\right\}$ associated to the objective properties $\left\{f_{\alpha}^{1}, \ldots, f_{\rho}^{n}\right\}$ that define a quantum object represented by the pure state $\psi=\left|f_{\alpha}^{1}, \ldots, f_{\rho}^{n}\right\rangle$, far from transforming 
the object into a different object, are just virtual phase transformations of the object. In particular, the angular orientations with respect to the $z$ axis of the objects represented by the quantum vectors $\left|\frac{1}{2}, \frac{1}{2}\right\rangle$ and $|1,1\rangle$ are not objective properties of the objects. Hence, the virtual transformations of these orientations generated by the action of $e^{i \hat{L}_{z} \theta}$ do not modify the objects as such.

It is worth stressing that the fact that phase transformations leave the objective properties of an object invariant does not mean that they are trivial transformations that cannot produce observable effects. Even if the overall orientation of a single die has no physical significance, a change in the orientation of a die in a system composed of two nested dice does modify the intrinsic structure of the whole system. Analogously, even if transformations of the absolute phase of a quantum object cannot have observable effects, we know from quantum mechanics that relative phases account for the quantum interference phenomena. Hence, the fact that the same abstract operation can be differently represented as effective transformations acting on quantum objects is far from being physically trivial, even if the corresponding phase transformations do not modify the objects as such.

In general, the proposed interpretative framework implies that quantum objects cannot be localized in both members of a complementary pair (such as $q$ and $p$ ). Indeed, the sharp localization on one of these variables entails that the other one is completely "phased out" by the phase transformations induced by the former. In Refs.[11, 12] we argued that Heisenberg uncertainty principle, far from resulting from an epistemic restriction to the amount of information an observer can have about an object, can be understood as a consequence of the circular imbrication between objective properties and non-objective phases established by the phase postulate. Indeed, the phase postulate entails that an objective property of an object must be invariant under the phase transformations induced by all the other objective properties of the same object. On the one hand, the fact that $\left\{f^{1}, \ldots, f^{n}\right\}$ is a complete set of commuting observables implies that the eigenvalues $\left\{f_{\alpha}^{1}, \ldots, f_{\rho}^{n}\right\}$ that define a quantum vector $\psi=\left|f_{\alpha}^{1}, \ldots, f_{\rho}^{n}\right\rangle$ are indeed invariant under the phase transformations generated by the operators $\left\{\hat{v}_{1}, \ldots, \hat{v}_{n}\right\}$. On the other hand, the variables that are canonically conjugated to the observables $\left\{f^{1}, \ldots, f^{n}\right\}$ cannot be objective properties of the same object, since they are not invariant under the phase transforma- 
tions generated by the operators $\left\{\hat{v}_{1}, \ldots, \hat{v}_{n}\right\}$. In particular, $q$ cannot be an objective property of an object defined by a sharp value of $p$, since $q$ is not invariant under the phase transformations induced by $p$. In this way, the phase postulate implies that a quantum object can only have $n$ sharp objective properties. By removing the dependence of the pre-quantum states on the surplus $n$ canonical variables, the introduction of a polarization formally implements the phase postulate. It is worth stressing that this restriction with respect to the overdetermined classical description is analogous to what happens in gauge theories: while the involution condition (7) implies that two first-class constraints are compatible only if they commute on the constraint surface, two observables $f$ and $g$ can define sharp objective properties of the same quantum object only if they have vanishing Poisson bracket (or, equivalently, if theirs associated quantum operators commute). This compatibility condition guarantees that the objective property $f_{0}$ defined by the observable $f$ (i.e. the eigenvalue $f_{0}$ of the quantum operator $\hat{v}_{f}$ ) is invariant under the phase transformations generated by $\hat{v}_{g}$ (and viceversa). The main consequence of this argument is that quantum vectors provide a complete description of all the objective (i.e. invariant under phase transformations) properties of quantum objects [11]. Hence, we can conclude that it is not the case that the quantum description of physical reality is incomplete, but rather that the classical description is overdetermined, since it does not take into account the difference between phase-invariant observables and phasedependent observables.

In this way, the factorization of the canonical quantization of a symplectic manifold $(P, \omega)$ in two independent stages matches the twofold construction of a quantum ontology by means of two independent postulates. On the one hand, the quantum postulate explains why a satisfactory ontology of physical objects requires to implement, by means of the pre-quantization formalism, an injective Lie algebra homomorphism between the Poisson algebra of observables $\mathcal{C}^{\infty}(P)$ and the Lie algebra of operators. On the other hand, the phase postulate explains why it is necessary to polarize the prequantum states, i.e. to select the pre-quantum states that are covariantly constant along a foliation of $(P, \omega)$ by Lagrangian submanifolds. 


\section{Conclusion}

In gauge theories, the existence of gauge symmetries reduce the number of physical degrees of freedom that are necessary to completely describe the state of a physical system. The heuristic idea of the present article is that the reduction in the number of observables that are necessary to describe a physical system from $2 n$ classical observables ( $q$ and $p$ ) to $n$ quantum observables $(q$ or $p)$ can be explained in an analogous way. Since this "quantum reduction" should be valid for both constrained and unconstrained Hamiltonian systems, it is necessary to introduce a universal symmetry principle different from that of gauge theories. In order to define such a symmetry principle, we proposed a general quantum ontology of physical objects. This quantum ontology can be understood as an extension of what we have called pre-ontology. According to the latter, an object is a multifaceted structure defined by a set of objective properties that are invariant under the phase transformations that interchange its different non-objective phases. We have then argued that the pre-ontology does not provide a general criterion for the determination of the phase group of a given object. The quantum ontology bypasses this flaw by supplementing the pre-ontology with two postulates, namely the phase postulate and the quantum postulate.

The phase postulates generalizes the gauge correspondence between firstclass constraints and gauge transformations to the observables of any unconstrained Hamiltonian system. This means that the observables that define the objective properties of the corresponding physical object induce-by means of their associated operators - the phase transformations between its non-objective phases. In this way, the phase postulate complements the standard correspondence objectivity = invariance by stating that the phase transformations under which the objective properties must be invariant are induced by the objective properties themselves. Far from producing a vicious circle, this virtuous circular relationship between objective properties and

phase transformations provides a conceptual explanation of the Heisenberg uncertainty principle. Indeed, the fact that different elements of a single phase orbit are "phase equivalent" reduces in half the number of observables that are necessary to completely describe the object. In other terms, the uncertainty principle formalizes the compatibility condition that results from the fact that an objective property has to be invariant under the phase transformations induced by all the other objective properties of the same 
object. Hence, asking after the objective position $q$ of an object with an objective momentum $p$ is as nonsensical as looking for the objective face of a die. In this way, the phase postulate explains why quantum mechanics does not admit sharp value attributions to both members of a complementary pair of observables. Moreover, this explanation does not appeal to a hypothetical epistemic restriction to the amount of information an observer can have about an object. Indeed, the phase postulate implies that quantum vectors, far from being states of incomplete knowledge, convey a complete description of all the objective properties of physical objects.

On the other hand, the quantum postulate establishes a faithful correspondence between the two roles played by physical observables in mechanics, namely (1) to assign numerical values to physical states, and (2) to induce transformations by means of their associated operators. According to the quantum postulate, the objective property $f_{0}$ defined by an observable $f$ faithfully specifies how the object transforms under the virtual phase transformations generated by the quantum operator $\hat{v}_{f}$. In this way, the quantum postulate provides a satisfactory interpretation of one of the essential features of mechanics, namely the correspondence between observables and operators. The important fact is that these two postulates cannot be implemented in the geometric arena of classical mechanics, i.e. symplectic geometry. Indeed, we argued that the quantum postulate and the phase postulate provide a satisfactory conceptual interpretation of the two independent stages of the geometric quantization formalism, namely the prequantization of a symplectic manifold and the polarization of the resulting pre-quantum states respectively.

In this way, the proposed interpretative framework sheds new light on two hallmarks of quantum mechanics, namely 1) that quantum mechanics does not admit sharp value attributions to both members of a complementary pair of observables, and 2) the existence of a faithful correspondence between (Poisson brackets of) observables and (commutators of) quantum operators. As we argued in Section VII, the main lesson of geometric quantization is that quantum mechanics can be essentially deduced from these two independent features. It is also worth stressing that this interpretative proposal, far from demanding philosophically costly hypothesis such as many-worlds, hidden variables or epistemic restrictions, only relies 1) on a particular interpretation of the standard correspondence between objectiv- 
ity and invariance under a symmetry group, and 2) a supplementation of such a correspondence with two independent postulates that match the two steps of the geometric quantization deduction of quantum mechanics.

It is also worth remarking that this interpretative framework permits us to propose a conjecture that can be mathematically explored. Indeed, the generalization of the gauge correspondence between first-class constraints

and gauge transformations to unconstrained Hamiltonian systems allows us to conjecture that the BRST formalism could be used for quantizing unconstrained Hamiltonian systems. More precisely, we briefly recalled in Section II that the action of the BRST operator encodes both the restriction to the constraint surface and the projection to the reduced phase space of the theory. Analogously, we can conjecture that quantum states can be obtained as solutions of a generalized eingevalue equation that has the same formal structure than the BRST condition $\Omega_{B R S T} \psi_{\text {phys. }}=0$ for physical states in gauge theories [25]. This means that this generalized eigenvalue equation should (1) fix the possible eigenvalues of the corresponding observable (e.g. $p$ ), and (2) phase out the canonically conjugated variable (e.g. q). If this conjecture were correct, then the polarization of the corresponding quantum vector, far from being an ad hoc condition on pre-quantum states as it is the case in geometric quantization, would be directly implemented by the generalized eigenvalue equation. From a conceptual point of view, the validity of such a conjecture would confirm that quantum mechanics does provide a generalization of the gauge correspondence between first-class constraints and symmetry transformations to unconstrained Hamiltonian systems. A forthcoming article will be devoted to the analysis of this conjecture [10].

\section{Acknowledgements}

This article stems from a talk given at the Perimeter Institute for Theoretical Physics in may 2009. I would like to thanks the members of the Quantum Foundations Group at the Perimeter Institute for their hospitality and helpful discussions.

\section{References}

[1] Abraham, R., \& Marsden, J. E. (1978). Foundations of Mechanics. (2nd ed.). Massachusetts: Addison-Wesley Publishing Company. 
[2] Arnold, V. I. (1989). Mathematical Methods of Classical Mechanics. New York: Springer.

[3] Arnowitt R., Deser S., \& Misner C. (1962). The dynamics of General Relativity, In L. Witten (Ed.), Gravitation: An Introduction to Current Research. New York: Wiley \& Sons.

[4] Auyang, S. Y. (1995). How is Quantum Field Theory Possible?. OxfordNew York: Oxford University Press.

[5] Bargmann, V. (1954). On Unitary Ray Representations of Continuous Groups. Annals of Mathematics, 59, 146.

[6] Born, M. (1998). Physical Reality. In E. Castellani (Ed.), Interpreting Bodies. Classical and Quantum Objects in Modern Physics (pp. 155-167). New Jersey: Princeton University Press.

[7] Brylinski, J.-L. (1993). Loop Spaces, Characteristic Classes, and Geometric Quantization. Progr. Math. 107. Boston: Birkhäuser Boston Inc.

[8] Busch, P., Lahti, P. J., \& Mittelsteadt, P. (1996). The Quantum Theory of Measurement. Heidelberg: Springer-Verlag.

[9] Butterfield, J. (2007). On Symplectic Reduction in Classical Mechanics. In J. Butterfield, \& J. Earman (Eds.), Philosophy of Physics, Handbook of the Philosophy of Science, Part A (pp. 1-131), Amsterdam: NorthHolland.

[10] Catren, G., \& Bennequin, D. (2011). BRST Cohomological Description of Geometric Quantization for Unconstrained Hamiltonian Systems. In preparation.

[11] Catren, G. (2009). Can Classical Description of Physical Reality be Considered Complete?. In M. Bitbol, P. Kerszberg, \& J. Petitot (Eds.), Constituting Objectivity: Transcendental Approaches of Modern Physics (pp. 375-386). The Western Ontario Series in the Philosophy of Science, Vol. 74. Berlin: Springer-Verlag.

[12] Catren, G. (2008). On Classical and Quantum Objectivity. Foundations of Physics, 38, 470-487. 
[13] Catren, G. (2008). Geometric foundations of classical Yang-Mills theory. Studies in History and Philosophy of Modern Physics, 39, 511-531.

[14] Catren, G., \& Devoto, J. (2008). Extended Connection in Yang-Mills Theory. Communications in Mathematical Physics, 284, 93-116.

[15] Castagnino, M., Catren, G. \& Ferraro, R. (2002). Time Asymmetries in Quantum Cosmology and Boundary Conditions to the Wheeler-DeWitt Equation. Classical and Quantum Gravity 19, No. 18, 4729-4746.

[16] Cassirer, E. (1944). The Concept of Group and the Theory of Perception. Philosophy and Phenomenological Research, Vol. 5, No. 1, 1-36.

[17] Cassirer, E. (1945). Reflections on the Concept of Group and the Theory of Perception. In E. Cassirer, Symbol, Myth and Culture. Essays and Lectures of Ernst Cassirer 1935-1945 pp. 271-291. London: New Haven, 1979.

[18] Castellani, E. (1998). Galilean Particles: An Example of Constitution of Objects. In E. Castellani (Ed.), Interpreting Bodies. Classical and Quantum Objects in Modern Physics (pp. 181-194). New Jersey: Princeton University Press.

[19] Castellani, E. (1995). Quantum Mechanics, Objects and Objectivity. In C. Garola \& A. Rossi (Eds.), The Foundations of Quantum Mechanics. Historical Analysis and Open Questions. Dordrecht: Kluwer.

[20] Debs, T., \& Redhead, M. (2007). Objectivity, Invariance, and Convention: Symmetry in Physical Science. Cambridge MA: Harvard University Press.

[21] Earman, J., \& Norton, J. (1987). What price spacetime substantivalism? The hole story. The British Journal for the Philosophy of Science, $38,515-525$.

[22] Fine, A. (1973). Probability and the Interpretation of Quantum Mechanics. The British Journal for the Philosophy of Science, Vol. 24, 1, $1-37$.

[23] French, S. (2001). Symmetry, Structure and the Constitution of Objects, available in http://philsci-archive.pitt.edu/archive/00000327/. 
[24] Guillemin, V., \& Sternberg, S. (1982). Geometric Quantization and Multiplicities of Group Representations. Inventiones Mathematicae, 67, 515-538.

[25] Henneaux, M., \& Teitelboim, C. (1994). Quantization of gauge systems. New Jersey: Princeton University Press.

[26] Ladyman, J. (1998). What is Structural Realism?. Studies in History and Philosophy of Science, Vol. 29, No. 3, 409424.

[27] Landsman, N. P. (2007). Between Classical and Quantum. In J. Butterfield \& J. Earman (Eds.), Philosophy of Physics, Handbook of the Philosophy of Science, Part A, Amsterdam: North-Holland, 417-553.

[28] Levy-Leblond, J.-M. (1967). Nonrelativistic Particles and Wave Equations. Communications in Mathematical Physics, 6, 286311.

[29] Misner C., Thorne K., \& Wheeler J. A. (1973). Gravitation, San Francisco: Freeman.

[30] Mittelstaedt, P., \& Weingartner, P. (2005). Laws of nature. BerlinHeidelberg: Springer-Verlag.

[31] Nozick, R. (2001). Invariances: The Structure of the Objective World. Cambridge, Mass.: Belknap Press of Harvard University Press.

[32] Kostant, B., \& Sternberg, S. (1987). Symplectic Reduction, BRS Cohomology and Infinite-Dimensional Clifford Algebras. Annals of Physics, 176, 49-113.

[33] Kostant, B. (1970). Quantization and Unitary representations. Lecture Notes in Mathematics, Vol. 170, Berlin-Heidelberg-New York: SpringerVerlag.

[34] Quine, W. V. (1969). Ontological Relativity and Other Essays. New York: Columbia University Press.

[35] Roberts, B. W. (2010). Group Structural Realism. The British Journal for the Philosophy of Science, 0, 1-23.

[36] Rovelli, C. (2004). Quantum gravity. Cambridge, MA: Cambridge University Press. 
[37] Souriau, J.-M. (1997). Structure of Dynamical Systems. A Symplectic View of Physics. Cambridge, MA: Birkhäuser Boston.

[38] Sternberg, S. (1995). Group Theory and Physics. Cambridge: Cambridge University Press.

[39] Weyl, H. (1931). The Theory of Groups and Quantum Mechanics. Trans. by H.P. Robertson. U.S.A.: Dover Publications, Inc.

[40] Weyl, H. (1952). Symmetry. New Jersey: Princeton University Press.

[41] Wigner, E. (1939). On Unitary Representations of the Inhomogeneous Lorentz Group. Annals of Mathematics, Vol. 40, No. 1, 149-204.

[42] Woodhouse, N. (1992). Geometric Quantization. (2nd ed.). Oxford: Oxford University Press. 